\title{
Aquatic toxicity integrated testing and assessment strategies (ITS) for difficult substances: case study with thiochemicals
}

\author{
Monika Nendza ${ }^{{ }^{*}}$ (D) and Jan Ahlers ${ }^{2}$
}

\begin{abstract}
Background: An Integrated Testing and Assessment Strategy (ITS) for aquatic toxicity of 16 thiochemicals to be registered under REACH revealed 12 data gaps, which had to be filled by experimental data. These test results are now available and offer the unique opportunity to subject previous estimates obtained by read-across (analogue and category approaches) to an external validation. The case study thiochemicals are so-called difficult substances due to instability and poor water solubility, challenging established ITS.

Results: The new experimental data confirm the previous predictions of acute aquatic toxicity with the new test results indicating a 2-5 times lower toxicity than previously predicted. The previous predictions thus are conservative and close to the new experimental results. The good agreement can be attributed to the fact that we had limited the extrapolations to narrow chemical groups with similar SH-group reactivities. The new experimental data further strengthen and externally validate the existing trends based on similarity in chemical structures, mode of action (MoA), water solubility and stability of source and target compounds in aquatic media. Based on the new experimental data, reliable revised PNECs could be derived and the REACH requirements for these thiochemicals are largely fulfilled. Appropriately adapted ITS are therefore able to reduce in vivo tests with fish even for difficult substances and replace them with alternative information.
\end{abstract}

Conclusions: Both experimental and alternative information for difficult substances such as thiochemicals that are rapidly transformed in water are subject to considerable uncertainty. For example, the use of either nominal, initial or time-weighted average concentrations contributes to the variability of aquatic toxicity data. In the case of these thiochemicals, a weight-of-evidence (WoE) approach to determining aquatic toxicity based on nominal and timeweighted average concentrations may be the most appropriate choice to reflect environmental conditions. Overall, uncertainties in historical test results and alternative information, here from read-across, have to be considered in relation to how much uncertainty is acceptable for environmental protection on the one hand and how much certainty is technically feasible on the other.

Keywords: Read-across, Mode of action (MoA), Evaluation of historical data, Chemical identity, Water solubility, Instability, Alternative information, Weight-of-evidence approach

*Correspondence: nendza@al-luhnstedt.de

${ }^{1}$ Analytical Laboratory, Bahnhofstr. 1, 24816 Luhnstedt, Germany

Full list of author information is available at the end of the article

\section{Background}

The European legislation on registration, evaluation, authorization and restriction of chemicals (REACH) aims to protect human health and the environment from hazards of industrial chemicals [1]. For this purpose, producers, importers and downstream users need to demonstrate the safe manufacture and use of chemicals 
throughout the supply chain. $\mathrm{REACH}$ requires hazard identification based on physicochemical, toxicological and ecotoxicological data with information requirements depending on the annual tonnage level. Similar procedures are used in many industrial countries including USA and Japan. The OECD chemicals program allows information exchange between the different regulatory frameworks. In particular, the OECD guidelines support comparable hazard assessments based on tests that are performed under standardized conditions.

REACH promotes alternative methods for the assessment of hazards of substances to avoid animal testing where possible. Integrated testing and assessment strategies (ITS) and weight-of-evidence (WoE) approaches [26] support a stepwise procedure (Box 1) for making best use of all available experimental data and to fill data gaps with alternative information like test results obtained with nonstandard organisms, deviations from standard test guidelines such as limit tests, in vitro assays, intra- or extrapolation from analogues (read-across), predictions from (quantitative) structure-activity relationships ((Q) SARs), and extrapolations from acute to chronic data and vice versa $[2,7,8]$. Alternative information is acceptable for REACH registrations if it is equivalent to the results that would be obtained by standard testing and adequate to draw conclusions for classification and labelling, PNEC derivation, and $\mathrm{PBT} / \mathrm{vPvB}$ assessment.

Both the determination of valid test data and even more so the proof of equivalence and adequacy of alternative information is a particular challenge for so-called difficult substances [9]. Their aquatic toxicity testing and assessment is often limited by losses of test substance from the test medium due to instability, volatility and/or adsorption. Poor water solubility is another confounding factor. The consequences are always the same: uncertainties about chemical identity and exposure concentration of the test item. These difficulties apply especially to historical data, where test guidelines and documentation frequently do not meet today's standards. Often the requirements of OECD Guidance Document 23 on Aquatic Toxicity Testing of Difficult Substances and Mixtures [9] are not fulfilled as the chemical was not identified as difficult substance in the past. More over, effect values may be difficult to compare if they are derived on the basis of nominal, initial or geometric (sometimes arithmetic) mean data. The testing difficulties also affect alternative methods such as read-across (analogue and category approach), ${ }^{1}$ which are generally based on existing

\footnotetext{
${ }^{1}$ For applying "read-across", we use the terms given by ECHA [10]: prerequisite is that the substances to be compared are likely to be similar or follow a regular pattern and thus may be considered as a group. The term "category approach" is used when read-across is employed between several similar substances and a trend can be established (trend analysis). The properties of the members of this group will either be similar or follow a regular pattern. The term "analogue approach" is used when the grouping is based on a very limited number of chemicals, where trends in properties are not apparent.
}

experimental data. Being aware of the uncertainties of the underlying historical data, that are propagated on to these estimates, we aim to make best use of all the existing information to avoid unnecessary animal testing.

In the present study, we investigate whether, despite the uncertainties mentioned above, it is possible to pragmatically but critically apply the ITS procedure to difficult substances according to the 3Rs principles (replacement, reduction and refinement of animal testing) [11-13] instead of standard testing requirements. Following the procedure laid out in Box 1, we will demonstrate that substitution of in vivo aquatic toxicity testing, especially with fish, by in silico methods is also possible for difficult substances. Our case study are two groups of thiochemicals, mercaptopropionates and mercaptans, which are relatively homogeneous substances with a limited number of functional groups, that are produced in the range of 1-100 t/year [14]. They are further processed or used directly in several downstream products (antioxidants in cosmetics, cleaners, polymers). Emerging fields are optical applications. These thiochemicals are considered difficult substances due to instability and/or very low water solubility, some of which show no effects up to the solubility limit.

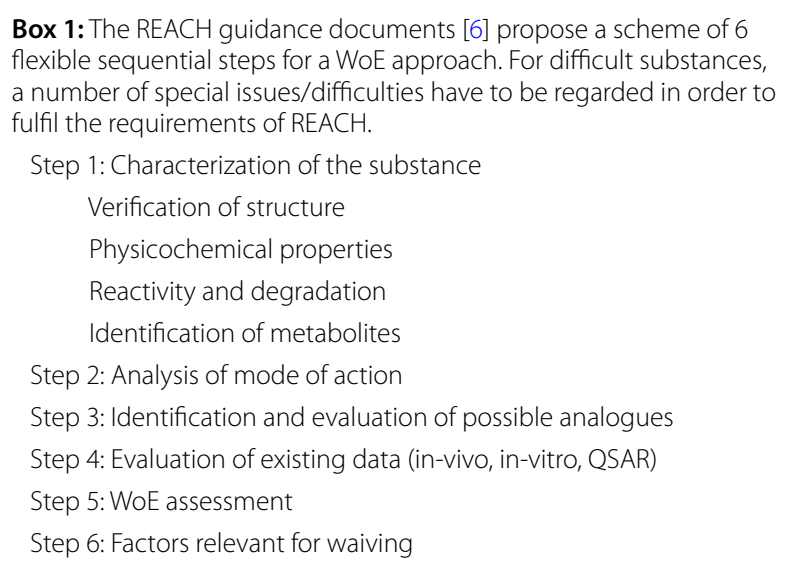

We discuss the following available information (Table 1):

1. Existing experimental data on thiochemicals: Some of these so-called historical data are relatively uncertain and often the methods have not been described adequately. However, they add information in the ITS procedure and might help to avoid testing especially with vertebrates if the combined information is equivalent to the results of standard testing.

2. Estimated data: Previous predictions were obtained by read-across applying category or analogue approaches [14, 15] after critical evaluation of the 


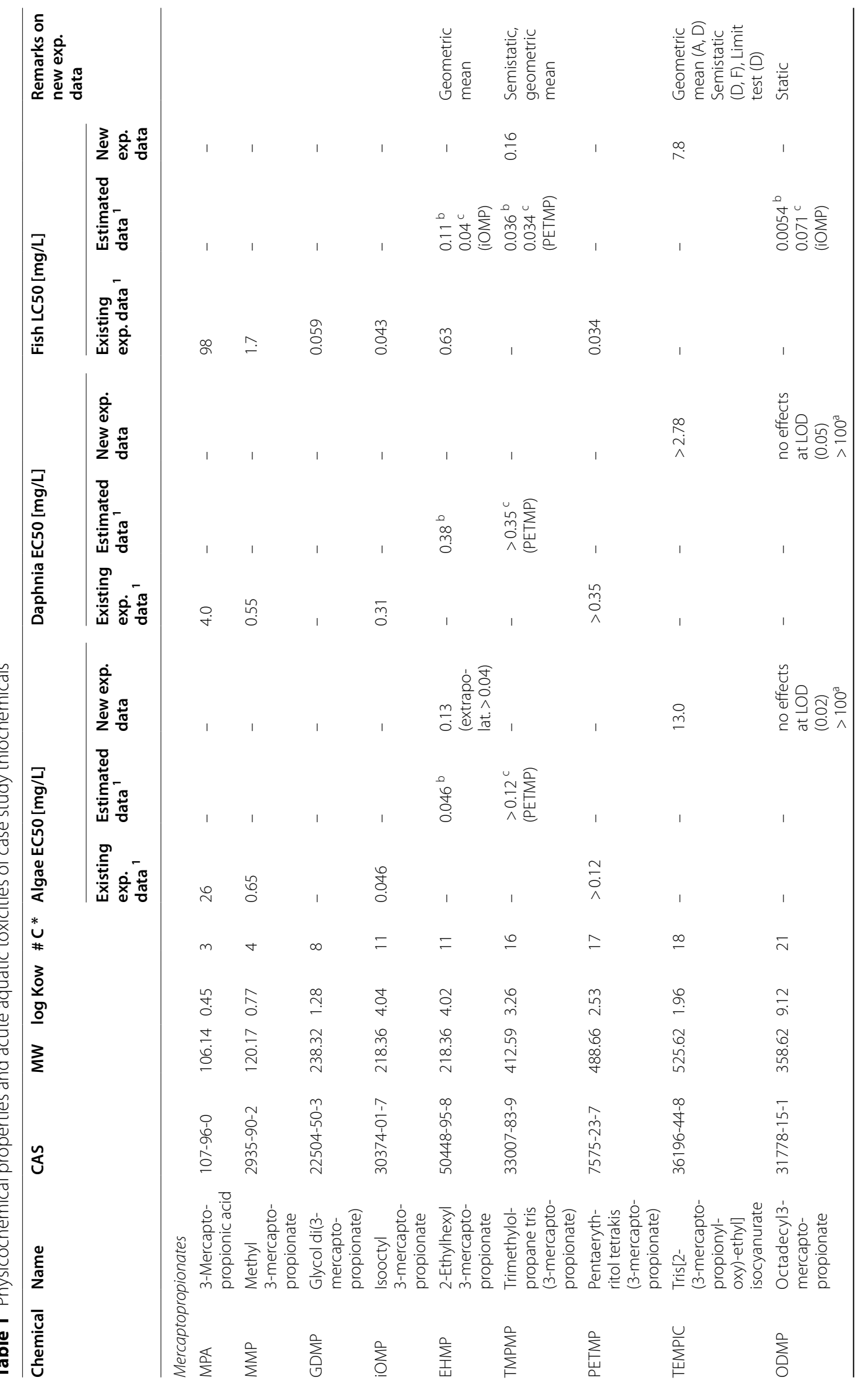




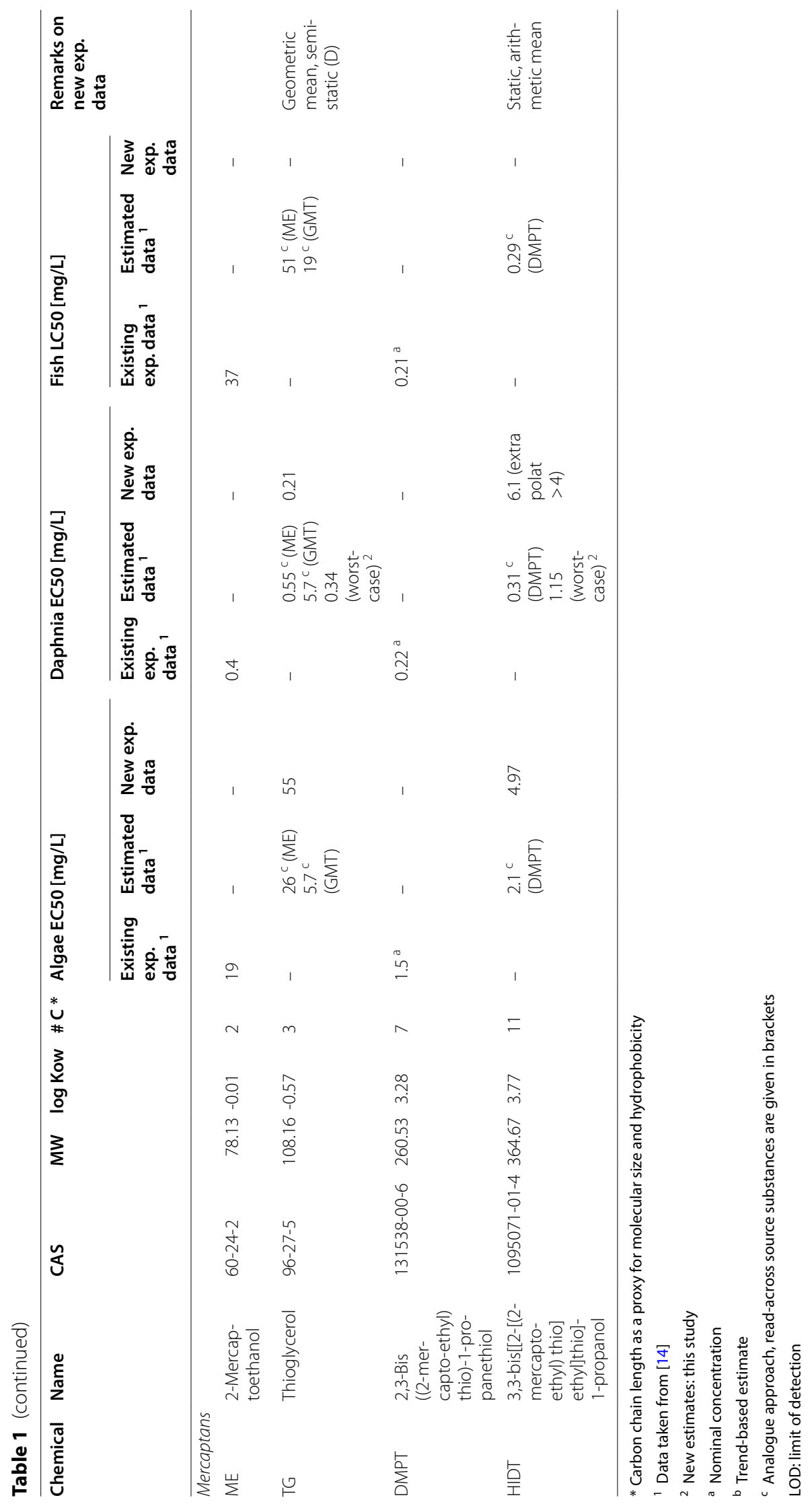


existing experimental data. This allowed data gaps to be filled for 13 mercaptopropionates and mercaptans to be registered in 2018 according to $\mathrm{REACH}$ requirements.

3. New experimental data: Remaining data gaps were closed by tests according to the relevant OECD test guidelines. The results of 11 new tests (5 algal, 4 daphnia and 2 fish tests) for mercaptopropionates and mercaptans are now available and can be used to consolidate the regulatory endpoints (PNECs) and to decide whether a substance has to be classified as dangerous for the environment. This will determine what additional information, for example results from chronic studies, may still be needed.

The information content of these data is considerably higher than that of historical data. However, instability and/or low solubility of the thiochemicals in the test solution lead to problems that need to be discussed.

It is the objective of this study to use the new data to evaluate our previous predictions, in the sense of an external validation, and to update the ITS described in [14]. Moreover, we discuss in the light of the relevant ECHA guidance $[6,7,10]$ under which conditions alternative information for difficult substances may be acceptable in the REACH registration process. We try to reveal that the idea behind ITS to use all available information in order to achieve a reliable hazard assessment is a better alternative for difficult substances compared to strictly following the test guidelines, especially in case of vertebrate tests.

\section{Material and methods}

The existing experimental, previously estimated and new experimental data for two groups of case study thiochemicals, mercaptopropionates and mercaptans, are presented in Table 1.

The octanol/water partition coefficients (log Kow) for the undissociated thiochemicals were calculated as the mean of the results from different independent algorithms (consolidated log Kow) [14, 15], including EpiSuite [16], ACD/Labs and ChemAxon from Chemspider [17], XLOGP and ALOGP from T.E.S.T. [18], Consensus, Read-across and LSER from ChemProp [19].

The new acute toxicity tests on algae [Pseudokirchneriella subcapitata, formerly known as Selenastrum capricornutum, and recently renamed as Raphidocelis subcapitata, $72 \mathrm{~h}$ growth rate (OECD 201)], daphnia [Daphnia magna, $48 \mathrm{~h}$ mobility (OECD 202)] and fish [rainbow trout (Oncorhynchus mykiss), 96-h mortality (OECD 203)] were performed according to the guidelines using good laboratory practice (GLP). For substances of low stability and very low water solubility, the recommendations of OECD 23 were applied [9]. The algae tests have been performed under static conditions, those with daphnia and fish due to the instability of these thiochemicals mostly semistatically (Table 1). Chemical concentrations in test samples were quantified by liquid chromatography with UV detection (EHMP, HIDT), MS/ MS detection (TMPMP, TEMPIC) or fluorescence detection (TG). For quantification of ODMP gas chromatography with MS detection was applied.

\section{Results and discussion}

Good environmental risk assessment practice for difficult substances is challenged by uncertainties about test item identity and exposure concentrations due to chemical instability and poor water solubility, or no measurable effects up to the limit of solubility. The assessment of our case study thiochemicals follows the flexible ITS framework (Box 1) based on integration of information from different sources and various aspects of uncertainty [6]. With the on-going filling of data gaps in the REACH registration process, we now have the opportunity to verify previous predictions with new measured values. This corresponds to an external validation and allows to learn which factors determine the quality of alternative information for difficult substances. In particular, we look at the influence of the chemical identity of the test item (see "Chemical identity" section), its physicochemical properties (see "Physicochemical properties" section), as well as the instability of the parent substance and effects of possible transformation products (see "Reactivity and degradation, identification of metabolites" section). As a key factor, we consider the analysis of the mode of action (see "Analysis of mode of action (MoA)" section). The evaluation of previous predictions against the new experimental data (see "Evaluation of previous predictions against new experimental data" section) leads to the revision and extension of trend analyses (see "Revision and extension of trend analyses" section). The new experimental results also have an influence on regulatory endpoints (PNEC) (see "Influence on regulatory endpoints (PNEC)" section). Finally, we discuss limitations regarding the practical aspects of carrying out valid tests with difficult test chemicals, and consider targeted modifications or additions to standard testing procedures according to OECD 23 [9]. The ITS improvements and adaptations (see "ITS improvements and adaptations" section) are dedicated to using alternative information whenever appropriate.

\section{Characterization of the test substance}

The identification of the actual test item is critical for high-quality test results used in scientific and regulatory assessments. Key is the central difficulty according 
to OECD 23 [9]: "Achieving, maintaining and measuring exposure concentrations". If little is known about chemical identity and concentration in the test system over time, a causal interpretation of the observed effects is hardly possible. In practice, this information is not available for many historical data. We will discuss when readacross and WoE may be feasible nevertheless.

\section{Chemical identity}

According to ECHA guidance [20], a well-defined substance is completely identified by its chemical composition, i.e. the chemical identity and the content of each constituent in the compound. For mono-constituent substances, verification of the structure relates to the main constituent $(\geq 80 \%)$ including the impurity profile. Multi-component substances have more than one main constituent $(\geq 10 \%$ and $<80 \%)$ as the result of a manufacturing process. According to the above definitions, the case study thiochemicals are well-defined mono-constituent substances with impurities, depending on composition of the starting materials in the production process. These include, for example, esters made with isomeric alcohols (e.g., isotridecyl alcohol, isooctyl alcohol), resulting in an isomeric mixture of esters. Reactions with polyvalent alcohols (4-valent: pentaerythritol, 3-valent: trimethylolpropane or glycerine) also lead to an ester mixture, for example 70\% 3-valent, 15\% 2-valent, 10\% monovalent; remainder: 5\% acid.

\section{Physicochemical properties}

Physicochemical properties determine the fate of a compound, its partitioning into a specific phase or compartment and largely influence the bioavailability to organisms which is decisive in bioaccumulation and toxicity tests. Information on physicochemical properties can support confidence in test results and underpin the basis for read-across in predicting environmental fate and effects.

The case study thiochemicals are relatively small molecules (average MW about 300, range 80-780) with mostly intermediate log Kow (average log Kow about 2.8, range -0.6-9). They are difficult substances because of their poor water solubility $(\mathrm{Sw})$ and instability, while adsorption and volatility are not major problems. In historical test protocols for thiochemicals, it has been reported that the total organic carbon of the test substance remained constant during the exposure time. The case study chemicals are rather insoluble in water, mostly $<50 \mathrm{mg} / \mathrm{L}$ [14]. However, reported concentrations in test media are often much lower than their solubility in pure water. Reasons for these discrepancies may be a combination of inappropriate methods and experimental deficiencies, in particular of historical studies, including, for example, insufficient length of solution preparation, poor use of filtration or stable unnoticed emulsions for low-solubility liquids. Some of these problems could have been avoided by using flow-through regimes or passive-dosing methods, but even despite the considerable analytical variability of the historical data on thiochemicals, it is at least possible to estimate the range of test concentrations that led to (no) observed effects.

\section{Reactivity and degradation, identification of metabolites}

Instability of test substances may reduce their exposure concentrations in bioassays, and at the same time result in mixtures of transformation products. Depending on Disappearance Time 50 (DT50) of the parent compound, different testing regimes are recommended [6], for example static, semi-static or flow-through. OECD 23 [9] provides advice to use geometric or arithmetic mean of concentrations at the beginning and the end of the experiment if $<80 \%$ of initial concentration remains at the end of the testing/renewal period.

Transformation of thiochemicals can occur by hydrolysis of the ester bond, forming aliphatic alcohols and (in case of mercaptopropionates) 3-mercaptopropionic acid. Oxidation with dissolved oxygen can produce disulfides within minutes to hours, but not always and not with all substances. The transformation products, such as alcohols and disulfides, are less reactive and arguably less toxic than the parent thiochemicals (see "Analysis of mode of action (MoA)" section). Under certain conditions, so-called glycolides/propionides can occur.

Transformation products with higher toxicity than the parent substances, for example by increasing their reactivity through additional reactive substructures, or by the formation of specific structures that can interact with sensitive receptors, are very unlikely for the quite small and chemically simple thiochemicals. Thus, it can be assumed that the parent thiochemicals cause the maximum (worst-case) toxicity of the test item and that any degradation leads to a decrease in toxicity.

Looking at the initial measured concentrations, it is remarkable that only $50 \%$ or less of the nominal concentrations of the parent substances were found in almost all tests. During the experiments, further decrease was observed in most assays. The extent of transformation can vary for the different chemical substances and between different bioassays. For example, the concentration of TG was moderately reduced in the static algae test (measured concentrations decreasing from 53 to 31\% of nominal), but strongly in the semi-static daphnia test 
(measured concentrations decreasing from $40 \%$ to $0.1 \%$ of nominal). The available information is not yet sufficient to systematically understand the factors causing problems with achieving, maintaining, and measuring exposure concentrations of difficult thiochemicals, and further studies are needed.

\section{Analysis of mode of action (MoA)}

In addition to similarities of the chemical structures and physicochemical properties, knowledge on similar MoA of source and target substances is needed to derive valid alternative information. MoA is the result of interactions between a chemical substance and biomolecular targets in exposed organisms. The determination of MoA is based on a common set of physiological and behavioural signs that characterize an adverse biological response [21]. Representing the cellular, physiological or organism-level effects of exposure to (a group of) chemicals, MoA is thus a descriptive, phenological term encompassing chemicals sharing certain effects. MoA grouping may also be based on an initial molecular interaction, for instance binding to membrane nucleophiles in the case of thiochemicals, with the remaining mechanisms by which these molecular initiating events (MIE) translate into organism-level effects being not necessarily known and can differ between chemicals with a similar MoA. The mechanism of action (MeoA) of a chemical, on the other hand, entails understanding the causal and temporal relationships between the steps leading to a particular effect, as well as the steps that lead to an effective dose of the chemical at the relevant biological target(s) of action [22]. Because knowledge of MeoA only exists for a limited number of compounds in ecotoxicology, the concept of MoA is used as a practical surrogate for grouping chemicals into a smaller number of more manageable groups, which, nevertheless, are still scientifically meaningful and practically useful [21,23].

MoA of thiochemicals has been considered in detail including characterization of MoA according to appropriate schemes and identification of structural alerts [14, 15]. According to the Verhaar scheme [24], the parent thiochemicals are class 3 type compounds (unspecific reactivity) with at least tenfold excess toxicity above baseline. In contrast, their transformation products such as alcohols and disulfides (see "Reactivity and degradation, identification of metabolites" section) are class 1 type compounds (narcosis or baseline toxicity).

In terms of functional similarity [25-28], the toxicological grouping of the thiochemicals is assumed to follow
Table 2 Chemical categories of case study thiochemicals

Chemical category

the chemical grouping (Table 2). ${ }^{2}$ Their toxicity is caused by the reactivity of the sulfur groups interacting with nucleophiles in membranes. The resulting reactive toxicity can significantly exceed so-called narcotic effects, i.e. the effects are much higher than estimated from thermodynamic partitioning into membranes according to log Kow-dependent baseline QSARs [28]. Comparison of the experimental acute toxicity of thiochemicals with baseline QSARs for algae [29], daphnia [30, 31], and fish [32, 33] revealed excess toxicities of more than one order of magnitude with distinct pattern for the different groups of thiochemicals [14]. Figure 1 illustrates the reactive excess toxicity of three groups of thiochemicals to fish. Similar results are obtained with algae and daphnia.

Quantitative differences in toxicity between the groups of thiochemicals may be due to differences in reactivity of the respective sulfur moiety, i.e. toxicodynamic differences. Within each group, the thiochemicals are different with regard to partitioning between biophases related to hydrophobicity, i.e. toxicokinetic differences. As a consequence, QSARs and read-across are limited to thiochemicals within the same group.

\section{Evaluation of previous predictions against new experimental data}

The quality of alternative information for difficult substances depends on issues discussed above, which are more or less addressed in the relevant guidelines, but are often neglected in practice or an adequate implementation is technically not feasible. Therefore, in the following, we will discuss the discrepancies between measured toxicities and alternative information, as well as the considerable uncertainties even of the best available experimental data for difficult substances that inevitably affect

\footnotetext{
2 The concept of functional similarity can support the MoA classification of chemicals by combining toxicological knowledge (which toxicity pathways are to be expected in which species under which exposure conditions) with chemical expertise (which parts of the chemical structures and physicochemical properties are involved in which interactions).
} 


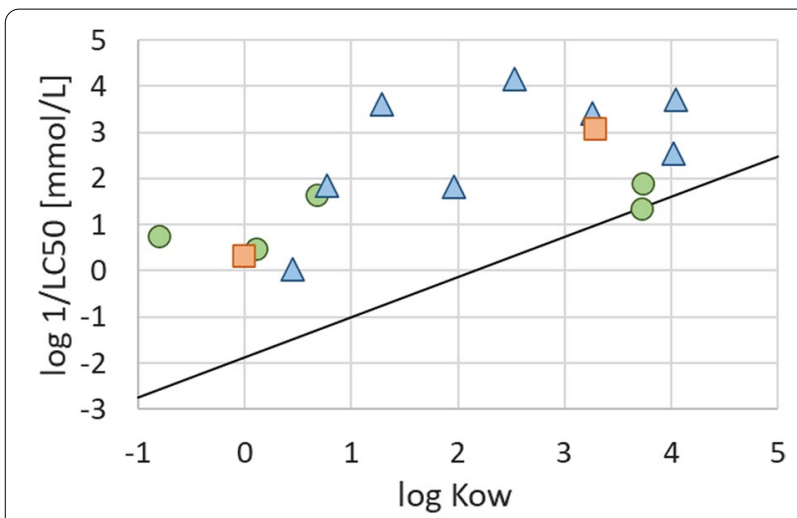

Fig. 1 MoA-related excess toxicity to fish of three categories of thiochemicals: thioglycolates (circles), mercaptopropionates (triangles) and mercaptans (squares) relative to the baseline QSAR by Könemann [32] (extended and updated with new experimental data from [15])

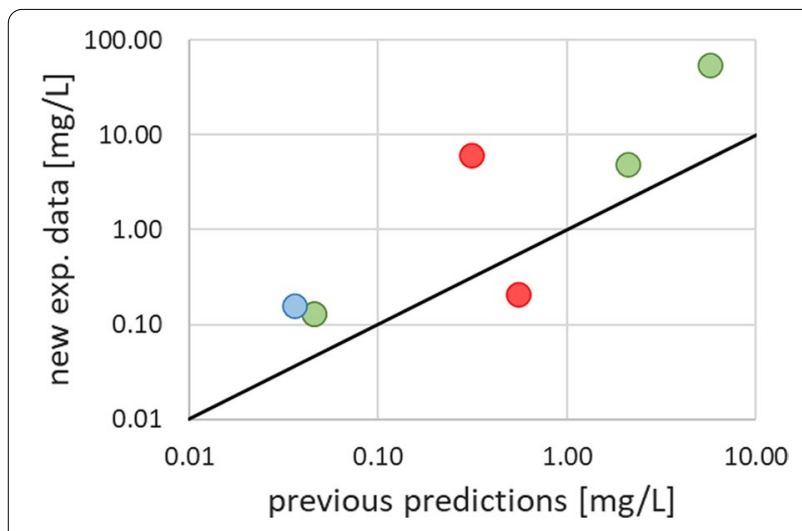

Fig. 2 Comparison of new test results with previous predictions [14] for algae (green circles), daphnia (red circles) and fish (blue circle)

the extrapolations derived from them by read-across. The errors propagated in this way can never be smaller than the uncertainties in the underlying test results.

Figure 2 presents a comparison of new measured aquatic toxicities for mercaptopropionates and mercaptans with those previously estimated but rated as not sufficiently reliable according to the REACH requirements (Table 1). Since 3 tests revealed no effects up to the limit of solubility and predictions were impossible for another 2 compounds, the diagram is limited to 6 data points from two groups of thiochemicals. The data points close to or above the 1:1 line illustrate that the previous predictions using read-across are conservative and do not underestimate the actual toxicities to significant extents. The only case of a lower estimate by a factor of two compared to the new test result is the daphnia toxicity of TG, which, however, is particularly uncertain due to the large decrease of the test item during exposure (the initial concentration was $40 \%$ of nominal and declined to $0.1 \%$ after $24 \mathrm{~h})$.

The good agreement of the algae toxicities (green circles) over more than 2 orders of magnitude confirm the conservatism of the previous predictions by analogue (TG and HIDT) and category approaches (EHMP). Obviously, the similar reactivities underlying the same MoA within the substance groups are a relevant basis for reliable read-across. In the case of fish toxicity (blue circle), the only new value for TMPMP $(0.16 \mathrm{mg} / \mathrm{L})$, which is 4 times higher than the almost identical estimates by analogue approach $(0.036 \mathrm{mg} / \mathrm{L})$ and trend analysis $(0.034 \mathrm{mg} / \mathrm{L})$, does not allow general conclusions to be drawn, but enables the extension of the corresponding trend (see "Revision and extension of trend analyses" section).

\section{Revision and extension of trend analyses}

In accordance with step 4 (Box 1 ), the new test results provide the opportunity to further elaborate the trend analyses for the two individual substance groups. Since most of the new data relate to mercaptopropionates, this is where most of the opportunities for improvement arise. This is exemplified by fish toxicity, for which there are two new experimental data. The extended data set (MPA, MMP, iOMP, EHMP, GDMP, TMPMP and PETMP) confirms the previously derived trend based on the carbon chain length (\# $\mathrm{C}$ ) as a proxy for molecular size and hydrophobicity (Fig. 3). Similar results are obtained based on log Kow or MW, but with somewhat lower $\mathrm{r}^{2}$, and are provided in Additional file 1.

Since TMPMP and PETMP, unlike the other mercaptopropionates, contain 3 and $4 \mathrm{SH}$ groups, respectively, the trends were calculated both with and without these two substances. As the correlations were almost identical (similar $r^{2}$ ), both thiochemicals remained included. Excluded is TEMPIC as it is very different from the other mercaptopropionates in its core structure and reactivity. Overall, this trend is now better substantiated due to the larger database and can thus provide more reliable estimates for further mercaptopropionates.

Regarding algal toxicity of mercaptopropionates, the new measured value for EHMP supports the previous trend based on only a few data, but is not sufficient to allow substantial improvements. The two new measured values for the algal toxicity of mercaptans (TG and HIDT) confirm the trend previously anticipated on the basis of only two substances (Fig. 4). 


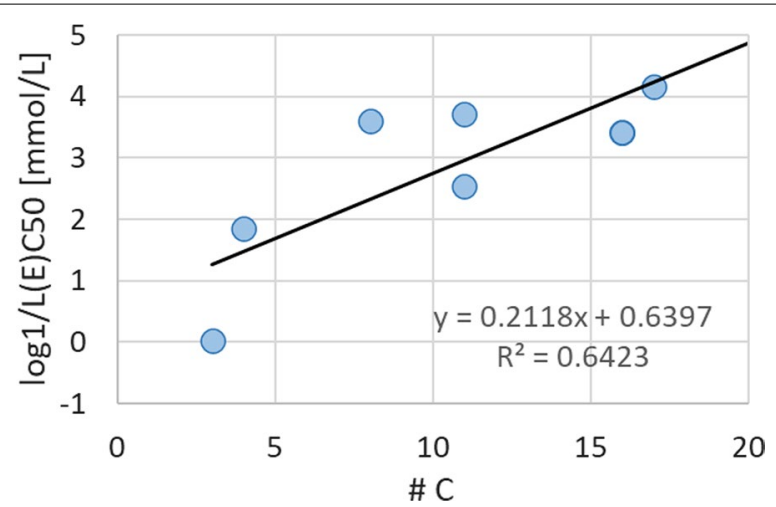

Fig. 3 Trend in acute fish toxicity of mercaptopropionates depending on the carbon chain length (\# C) as a proxy for molecular size and hydrophobicity, the new value for TMPMP (\# C = 16) fits very well. Corresponding (similar) plots based on log Kow or MW are available in Additional file 1.

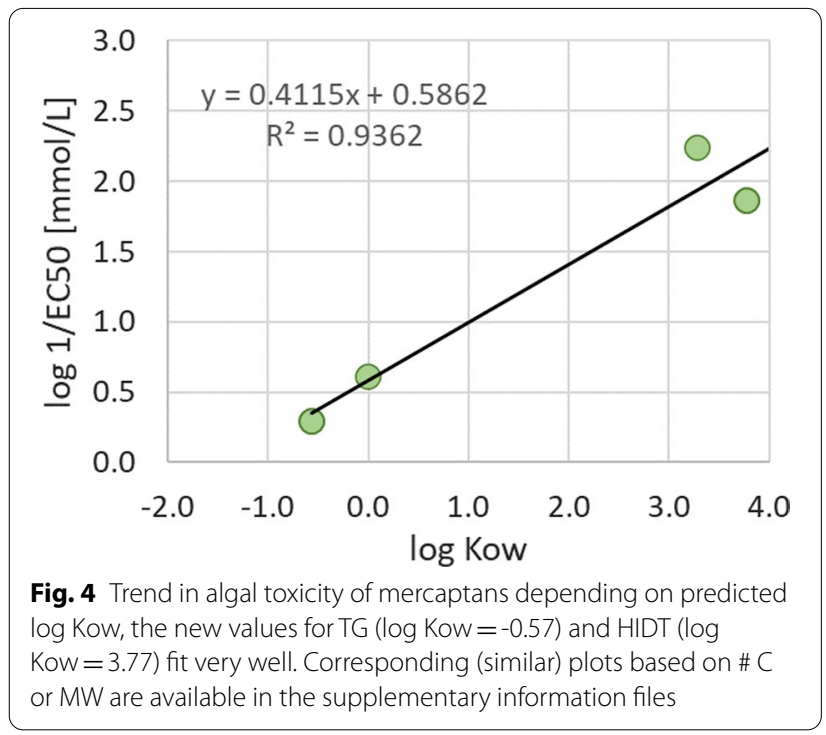

No trend of increasing or decreasing daphnia toxicity of the mercaptans with \# C, log Kow or MW can be observed, rather we see similar molar effect concentrations over the entire range of substances (Fig. 5). The similar reactivities causing similar toxicities suggest realistic worst-case toxicity estimates in daphnids of about $0.003 \mathrm{mmol} / \mathrm{L}$ for this substance group.

The good agreement of the new experimental data with our previous estimates using trend analyses consequently leads to further strengthening of the trends and thus to their external validation. From these findings, we conclude that even trends based on limited data can provide relevant alternative information also for difficult substances if similarity in MoA, chemical structures and

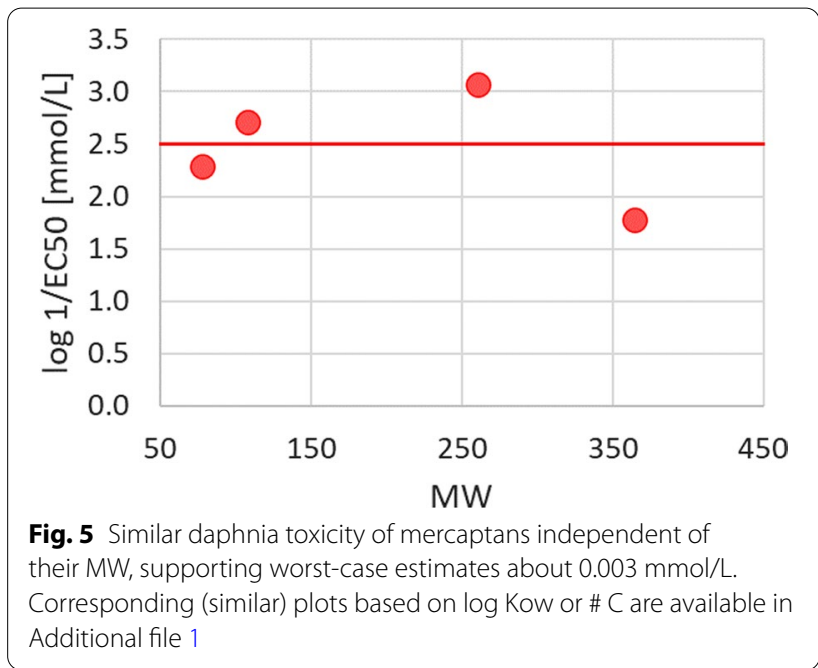

behaviour in aquatic media are adequately considered in terms of water solubility and stability of source and target compounds.

\section{Influence on regulatory endpoints (PNEC)}

In a WoE approach (step 5, Box 1) the new test results (Table 1) are used to derive valid PNECs for five substances, which replace uncertain ones (TMPMP, EHMP, HIDT, TG) or fill the gaps (TEMPIC) (Table 3).

The new data caused changes of $C \& L$ as hazardous for the environment only for HIDT that is now classified as category $\mathrm{C} 2$ (toxic to aquatic life with long lasting effects) instead of the former uncertain classification C1 (very toxic to aquatic life with long lasting effects). Regarding the requirements of REACH for aquatic toxicity, the following conclusions can be drawn for the case study chemicals:

- For EHMP the uncertain estimate for algae is replaced by a valid test result: no further information requirements.

- For TMPMP a valid acute fish test and reliable estimates for algae and daphnia are available: no further information requirements.

- For TEMPIC test results with algae, daphnia and fish are available: no further information requirements.

- For TG two uncertain estimates (algae and daphnia) are replaced by test results. The PNEC is based on the daphnia test (geometric mean) and is rather uncertain but conservative due to the decline of test concentration to $0.1 \%$ of the nominal concentration within $24 \mathrm{~h}$. In case the resulting PEC/PNEC ratio indicates a risk, the daphnia test should be repeated under flow-through conditions. Read-across (ana- 
Table 3 Revision of PNECs for mercaptopropionates and mercaptans

\begin{tabular}{llll}
\hline Name & $\operatorname{PNEC}_{\text {revised }}(\boldsymbol{\mu g} / \mathbf{L})$ & $\operatorname{PNEC}_{\text {previous }}(\boldsymbol{\mu g} / \mathbf{L})$ & Basis for revised PNEC \\
\hline EHMP & 0.13 & 0.05 & Test result for algae $(0.13 \mathrm{mg} / \mathrm{L})$ replaces former estimation \\
TMPMP & 0.16 & 0.04 & New test result for fish $(0.16 \mathrm{mg} / \mathrm{L})$ replaces the former prediction \\
TEMPIC & 7.8 & - & New test result for fish $(7.8 \mathrm{mg} / \mathrm{L})$ \\
TG & 0.21 & 0.55 & Test result for daphnia $(0.21 \mathrm{mg} / \mathrm{L})$ replaces former prediction \\
HIDT & 5.0 & 0.29 & Test result for algae $(4.97 \mathrm{mg} / \mathrm{L})$ replaces former predictions \\
\hline
\end{tabular}

logue approach) of the fish toxicity is possible from 2-mercaptoethanol, being a substructure of TG, as well as from GMT. Both extrapolations indicate that TG is less toxic to fish than to daphnia. In order to avoid vertebrate testing, an acute fish test should only be considered if PEC/PNEC $>1$.

- For HIDT two uncertain estimates (algae and daphnia) are replaced by tests leading to an about 20-fold increase of the PNEC. The estimated fish toxicity (analogue approach) is of a similar magnitude to that of daphnia. A fish test should only be performed in case of the PEC/PNEC ratio $>1$ in order to avoid vertebrate testing. Alternatively, a limit fish test at a concentration of $5 \mathrm{mg} / \mathrm{L}$ ( $\mathrm{EC}_{50}$ of algae) is proposed.

Since our previous estimates were generally conservative and the new experimental data confirm lower toxicities, most PNECs could be adjusted upwards. From this observation, we conclude that with relatively few additional tests reliable PNECs can also be obtained for difficult substances, and hence vertebrate studies can be avoided as much as possible.

\section{ITS improvements and adaptations}

$\mathrm{REACH}$ promotes the application of integrated testing and assessment strategies. By combining testing and nontesting information in a WoE approach, an enhancement of the reliability of hazard assessment and at the same time reduction of (vertebrate) testing is intended. Such a procedure is especially important for difficult substances with considerably uncertain test results.

The regulatory acceptance of alternative information strongly depends on the extent to which its relevance and adequacy is communicated. Especially for historical data of the case study thiochemicals, information on the chemical identity and exposure concentration of the test item, i.e. the parent compound and any degradation products over time, are not always available.

The question arises, which strategy is most appropriate to assess the aquatic toxicity of instable substances like thiochemicals, considering what happens in the environment. In accordance with OECD 23 [9], in most cases the aquatic toxicity was determined via geometric mean of nominal/initial concentration and remaining concentration at the end of the test or in semi-static tests after $24 \mathrm{~h}$. In several cases, the concentration at the end of the test was well below $1 \%$ of the nominal or initial ones or even below the limit of detection. Applying the geometric mean approach is formally correct, but does not reflect the situation under environmental conditions and yields therefore uncertain results. For example, if a nominal concentration of $100 \mathrm{mg} / \mathrm{L}$ decreases to $1 \mathrm{mg} / \mathrm{L}$ during time of exposure, a geometric mean $\mathrm{EC}_{50}$ of $10 \mathrm{mg} / \mathrm{L}$ is derived and considered as average concentration being responsible for the toxicity of the compound. However, it additionally reflects $90 \mathrm{mg} / \mathrm{L}$ of the transformation product(s). Therefore, it can at best be accounted as a (however very uncertain) result, if it is assumed that the transformation product is not toxic at all or disappears from the test medium or the environmental compartment during the course of exposure. Another source of uncertainty has to be considered, if at the end of the test the concentration of the substance tested is lower than the limit of detection. In this case, the sensitivity of the analytical method determines the toxicity: the more sensitive the analytics, the lower the time-weighted average concentrations and thus the calculated $\mathrm{EC}_{50}$ values.

As discussed above, it can be assumed that the reactivity of the degradation products of the case study thiochemicals is reduced by hydrolysis or oxidation to disulfides and thus their toxicity decreases. The following alternatives have to be discussed in order to reflect the situation in the environment:

1. The transformation product(s) exhibit the same aquatic toxicity as the parent substance. Calculation of the aquatic toxicity on the basis of nominal concentration is the adequate approach and reflects the situation in the environment. Time-weighted average concentrations of the parent compound would overestimate the hazard of the mixture actually present.

2. The transformation products are non-toxic or disappear from the aquatic compartment. Under these conditions, the aquatic toxicity should be determined from the time-weighted average concentration. 
Nominal concentrations would underestimate the aquatic toxicity.

3. The transformation products of the case study thiochemicals are less, but still considerably toxic due to hydrolysis and/or oxidation with the consequence that time-weighted average concentrations overestimate and nominal concentrations underestimate aquatic toxicity. Therefore, in a WoE approach (Step 5 of box 1 ) both estimates in combination with additional information should be used for hazard identification. For risk assessment such an approach presents greater confidence, if both estimates lead to a $\mathrm{PEC} / \mathrm{PNEC}$ ratio $<1$ or $>1$. In the other case more information on the time-course and/or mechanism of transformation as well as a more intensive investigation of the aquatic toxicity of the reaction products has to be obtained.

Additional uncertainty arises when organic solvents are used due to low solubility or when the WAF procedure is applied. In these cases, the exact nominal concentrations in test solution are not known and the initial measured concentration should be used instead.

For better regulatory acceptance of alternative information, the conservatism of estimates can be furthermore verified by bridging studies. Their basis is a similar unspecific reactive MoA of these thiochemicals in most species, which is then used as the decisive similarity characteristic. To begin with, estimates for algal, daphnia and fish toxicity are obtained using comparable methods, e.g., read-across preferably from the same source substance. Then experimental data are obtained for algae and daphnia, the so-called bridging studies, and compared with the estimated values. If there is sufficient agreement, it can be assumed that the prerequisites for a read-across for fish are also fulfilled and thus the fish test can be waived.

The problems with difficult substances discussed above show that it is often impossible to achieve the desired quality of test results. Repetition of tests without applying ITS concepts does not usually lead to more confidence. When ambiguities remain, these lead to the question of how much uncertainty in the hazard assessment of chemicals may be acceptable, or has to be tolerated, in particular when the technical feasibility of studies is limited. At the same time, the issue of realism (what is (supposed to be) in the test vessels versus what is in the environment) emerges. To balance the need for sustainable protection of human health and the environment against hazard and risk assessment reality, it is important to consider the magnitude of potential impacts of a substance and their possible persistence.

\section{Conclusions}

Compliance with $\mathrm{REACH}$ requirements for the case study thiochemicals was largely achieved with 11 new tests (5 algal, 4 daphnia and 2 fish tests) in addition to historical data and alternative information (trend analyses, analogue approach) based on these data. Only for TG and HIDT additional fish tests should be considered if the PEC/PNEC ratio is $>1$.

The external validation proved our previous estimates to be conservative and therefore protective for the environment. In the present study, this allowed us to further improve ITS components such as category and analogue approaches. Their inevitable uncertainties may be (partly) compensated with other testing and non-testing information with the consequence that vertebrate testing can be avoided in several cases. The confidence in alternative information on difficult substances depends on detailed and comprehensible documentation, which particularly regards the MoA of both the parent substance and transformation products in the case of aquatic toxicity. Since the WoE procedure and its documentation can be very time-consuming, especially for difficult substances the performance of tests with algae and daphnia is clearly recommended, and also justified on ethical and economic grounds. These bridging studies can then be used to support the replacement of studies with fish.

For unstable substances, which undergo a rapid transformation in water, it is important to assess their toxicities on the basis of nominal or initial as well as on time-weighted measured concentrations in order to achieve in a WoE approach the best estimates for hazard and risk assessments. With regard to the structure and mode of action of possible transformation products, this approach reflects more realistically the situation in the environment, which is consistent with the approach recommended for multi-component-substances or UVCB.

\section{Abbreviations \\ C\&L: Classification and labelling; DT50: Disappearance time 50\%; ECHA: European Chemicals Agency; ITS: Integrated Testing and Assessment Strategy; LOD: Limit of detection; log Kow: Octanol/water partition coefficient; MeoA: Mechanism of action; MoA: Mode of action; OECD: Organisation for Economic Co-operation and Development; PBT/VPVB: Persistent, bioaccumulative, toxic/ very persistent, very bioaccumulative; PEC: Predicted environmental concen- tration; PNEC: Predicted no effect concentration; 3Rs: Replacement, Reduction, and Refinement of animal testing; REACH: European legislation on registration, evaluation, authorization and restriction of chemicals; (Q)SAR: (Quantitative) structure-activity relationships; Sw: Water solubility; UVCB: Substance of Unknown or Variable composition, Complex reaction products or Biological materials; WoE: Weight-of-evidence.}

\section{Supplementary Information}

The online version contains supplementary material available at https://doi. org/10.1186/s12302-022-00591-6. 
Additional file 1. Trends in acute aquatic toxicities of mercaptopropionates and mercaptans based on the carbon chain length (\# C) as a proxy for molecular size and hydrophobicity, log Kow and MW.

\section{Acknowledgements}

We thank Bruno Bock Thiochemicals, Eichholzer Straße 23, 21436 Marschacht, Germany, for providing results of aquatic toxicity tests for the case study chemicals and Dirk Schwartz, Bruno Bock Thiochemicals, for valuable discussions.

\section{Authors' contributions}

$\mathrm{MN}$ and JA contributed to the design and implementation of the research, to the analysis of the results and to the writing of the manuscript. Both authors read and approved the final manuscript.

\section{Funding}

The authors did not receive any funding for this research work.

This article belongs to a series of contributions submitted from members of the "Division of Environmental Chemistry and Ecotoxicology" of the "German Chemical Society (GDCh)".

\section{Availability of data and materials}

All data generated or analysed during this study are included in this published article and in additional files.

\section{Declarations}

Ethics approval and consent to participate

Not applicable.

\section{Consent for publication}

Not applicable.

\section{Competing interests}

M.N. and J.A. have previously worked as consultants for Bruno Bock Thiochemicals, resulting in the published previous predictions and the proposals for additional new experimental studies used by Bruno Bock Thiochemicals for REACH registrations. The scientific evaluation and interpretation of the data presented here is the sole responsibility of M.N. and J.A. and received no funding. There is no conflict of interest that could inappropriately influence or bias the content of the paper.

\section{Author details}

1Analytical Laboratory, Bahnhofstr. 1, 24816 Luhnstedt, Germany. ${ }^{2}$ Berlin, Germany.

Received: 11 November 2021 Accepted: 16 January 2022

Published online: 22 February 2022

\section{References}

1. European Commission (2006) REGULATION (EC) No 1907/2006 OF THE EUROPEAN PARLIAMENT AND OF THE COUNCIL of 18 December 2006 concerning the Registration, Evaluation, Authorisation and Restriction of Chemicals (REACH), establishing a European Chemicals Agency, amending Directive 1999/45/EC and repealing Council Regulation (EEC) No 793/93 and Commission Regulation (EC) No 1488/94 as well as Council Directive 76/769/EEC and Commission Directives 91/155/EEC, 93/67/EEC, 93/105/EC and 2000/21/EC. European Commission, Brussels, Belgium

2. Ahlers J, Stock F, Werschkun B (2008) Integrated testing and intelligent assessment - new challenges under REACH. Environ Sci Pollut Res 15:565-572

3. Lombardo A, Roncaglioni A, Benfenati E, Nendza M, Segner H, Jeram S, Paunée E, Schüürmann G (2014) Optimizing the aquatic toxicity assessment under REACH through an integrated testing strategy (ITS). Environ Res 135:156-164
4. Rovida C, Alépée N, Api AM, Basketter DA, Bois FY, Caloni F, Corsini E, Daneshian M, Eskes C, Ezendam J, Fuchs H, Hayden P, Hegele-Hartung C, Hoffmann S, Hubesch B, Jacobs MN, Jaworska J, Kleensang A, Kleinstreuer N, Lalko J, Landsiedel R, Lebreux F, Luechtefeld T, Locatelli M, Mehling A, Natsch A, Pitchford JW, Prater D, Prieto P, Schepky A, Schüürmann G, Smirnova L, Toole C, van Vliet E, Weisensee D, Hartung T (2015) Integrated Testing Strategies (ITS) for Safety Assessment. Altex 32(1):25-40

5. Scientific Committee EFSA, Hardy A, Benford D, Halldorsson T, Jeger MJ, Knutsen HK, More S, Naegeli H, Noteborn H, Ockleford C, Ricci A, Rychen G, Schlatter JR, Silano V, Solecki R, Turck D, Benfenati E, Chaudhry QM, Craig P, Frampton G, Greiner M, Hart A, Hogstrand C, Lambre C, Luttik R, Makowski D, Siani A, Wahlstroem H, Aguilera J, Dorne JL, Dumont AF, Hempen M, Martínez SV, Martino L, Smeraldi C, Terron A, Georgiadis $\mathrm{N}$, Younes M (2017) Guidance on the use of the weight of evidence approach in scientific assessments. EFSA J 15:8. https://doi.org/10.2903/j. efsa.2017.4971

6. ECHA (2017) Guidance on information requirements and chemical safety assessment Chapter R.7b: Endpoint specific guidance. https://op.europa. eu/en/publication-detail/-/publication/cf4517df-6063-11e7-954d-01aa7 5ed71a1/language-en.

7. ECHA (2016) Practical guide How to use alternatives to animal testing to fulfil your information requirements for REACH registration.

8. Ahlers J, Riedhammer C, Vogliano M, Ebert R-U, Kühne R, Schüürmann G (2006) Acute to chronic ratios in aquatic toxicity — variation across trophic levels and relationship with chemical structure. Environ Toxicol Chem 25(11):2937-2945. https://doi.org/10.1897/05-701R.1

9. OECD (2019) Guidance document on aqueous-phase aquatic toxicity testing of difficult test substances. Series on Testing \& Assessment, Paris

10. ECHA (2017) Read-Across Assessment Framework (RAAF).

11. Burden N, Benstead R, Clook M, Doyle I, Edwards P, Maynard SK, Ryder K, Sheahan D, Whale G, van Egmond R, Wheeler JR, Hutchinson TH (2016) Advancing the $3 R$ in regulatory ecotoxicology: A pragmatic cross-sector approach. Integr Environ Assess Manag 12(3):417-421. https://doi.org/10. 1002/ieam.1703

12. Jeram S, Riego Sintes JM, Halder M, Baraibar Fentanes J, Sokull-Klüttgen $B$, Hutchinson TH (2005) A strategy to reduce the use of fish in acute ecotoxicity testing of new chemical substances notified in the European Union. Reg Toxicol Pharmacol 42:218-222

13. Russell WMS, Burch RL (1959) The Principles of Humane Experimental Techniques. Methuen, London, UK

14. Ahlers J, Nendza M, Schwartz D (2019) Environmental hazard and risk assessment of thiochemicals. Application of integrated testing and intelligent assessment strategies (ITS) to fulfil the REACH requirements for aquatic toxicity. Chemosphere 214:480-490. https://doi.org/10.1016/j. chemosphere.2018.09.082

15. Nendza M, Ahlers J, Schwartz D (2020) QSARs and Read-Across for Thiochemicals: A Case Study of Using Alternative Information for REACH Registrations. In: Roy K (ed) Ecotoxicological QSARs. Springer, New York, pp 545-560

16. US EPA (2012) KOWWIN v1.68 from EPISuite, Estimation Programs Interface Suite ${ }^{\mathrm{TM}}$ for Microsoft ${ }^{\circledR}$ Windows, v 4.11. United States Environmental Protection Agency, Washington, DC, USA. https://www.epa.gov/tscascreening-tools/epi-suitetm-estimation-program-interface.

17. Royal Society of Chemistry (2018) ChemSpider. http://www.chemspider. com.

18. US EPA (2012) T.E.S.T. v4.1. http://www.epa.gov/nrmrl/std/qsar/qsar.html.

19. ChemProp (2019) http://www.ufz.de/ecochem/chemprop.

20. ECHA (2017) Guidance for identification and naming of substances under REACH and CLP. doi:https://doi.org/10.2823/538683

21. Vonk JA, Benigni R, Hewitt M, Nendza M, Segner H, van de Meent D, Cronin MTD (2009) The use of mechanisms and modes of toxic action in integrated testing strategies. ATLA 37(5):557-571

22. Borgert CJ, Quill TF, McCarty LS, Mason AM (2004) Can mode of action predict mixture toxicity for risk assessment? Toxicol Appl Pharmacol 201(2):85-96. https://doi.org/10.1016/j.taap.2004.05.005

23. McCarty LS, Borgert CJ (2017) Comment on "Mode of Action (MOA) assignment classifications for ecotoxicology: an evaluation of approaches." Environ Sci Technol 51(22):13509-13510. https://doi.org/10. 1021/acs.est.7b04967 
24. Verhaar HJM, van Leeuwen CJ, Hermens JLM (1992) Classifying environmental pollutants. 1: structure-activity relationships for prediction of aquatic toxicity. Chemosphere 25:471-491

25. Nendza M, Müller M, Wenzel A (2014) Discriminating toxicant classes by mode of action: 4 Baseline and excess toxicity. Environ Res 25(5):393-405. https://doi.org/10.1080/1062936X.2014.907205

26. Nendza M, Wenzel A (2006) Discriminating toxicant classes by mode of action: 1. (Eco)toxicity profiles. Environ Sci Pollut Res 13:192-203

27. Nendza M, Müller M (2000) Discriminating toxicant classes by mode of action: 2 Physico-chemical descriptors. Quant Struct-Act Relat 19:581-598

28. Nendza M, Müller M, Wenzel A (2017) Classification of baseline toxicants for QSAR predictions to replace fish acute toxicity studies. Environ Sci Process Impacts 19(3):429-437. https://doi.org/10.1039/C6EM00600K

29. Shigeoka T, Sato Y, Takeda Y, Yoshida K, Yamauchi F (1988) Acute toxicity of chlorophenols to green algae, Selenastrum capricornutum and Chlorella vulgaris, and quantitative structure-activity relationships. Environ Toxicol Chem 7:847-854

30. Hermens JLM, Canton H, Janssen P, de Jong R (1984) Quantitative structure-activity relationships and toxicity studies of mixtures of chemicals with anaesthetic potency: acute lethal and sublethal toxicity to Daphnia magna. Aquat Toxicol 5:143-154

31. Deneer JW, van Leeuwen CJ, Maas-Diepeveen JL, Hermes JLM (1989) QSAR study of the toxicity of nitrobenzene derivatives towards Daphnia magna, Chlorella pyrenoidosa and Photobacterium phosphoreum. Aquat Toxicol 15:83-98

32. Könemann H (1981) Quantitative structure-activity relationships in fish toxicity studies. Part I: Relationship for 50 industrial pollutants. Toxicology 19:209-221

33. Nendza M, Russom CL (1991) QSAR modeling of the ERL-D Fathead minnow acute toxicity database. Xenobiotica 21:147-170

\section{Publisher's Note}

Springer Nature remains neutral with regard to jurisdictional claims in published maps and institutional affiliations.

\section{Submit your manuscript to a SpringerOpen ${ }^{\circ}$ journal and benefit from:}

- Convenient online submission

- Rigorous peer review

- Open access: articles freely available online

- High visibility within the field

- Retaining the copyright to your article

Submit your next manuscript at $\boldsymbol{\nabla}$ springeropen.com 